\title{
Small spinal fractures in back pain patients
}

\author{
HEATHER SIMS-WILLIAMS, MALCOLM I. V. JAYSON, AND \\ HIRAM BADDELEY
}

From the Department of Medicine, University of Bristol, and Department of Radio-diagnosis, Bristol Royal ${ }_{\circledast}$ Infirmary

SUMMARY Small fractures in the posterior elements of the spine were identified by stereoscopic radiography in 7 patients suffering from back pain. The clinical data on these 7 patients are presented.

Pain in the lumbar region is the commonest rheumatological disability. It is possible to identify the causes of the pain in some patients, but in many the underlying pathogenic mechanism is uncertain. Radiological changes may be found in the lumbar spine, but their significance is uncertain. It seems better to label these patients as having 'nonspecific back pain', a term which carries no pathological implications, rather than apply diagnostic terms which have no proven basis.

Gross fractures of the lumbar spine following trauma produce acute back pain and are easily recognised. At the other extreme, microfractures of trabeculae may occur along lines of stress (VernonRoberts and Pirie, 1973). They have been found in pathological studies, but cannot be identified in life. Their clinical significance is uncertain, but they could be responsible for acute episodes of back pain which settle as the fracture heals. The use of the Zeiss stereoplotter (Jacoby et al., 1976) enables us to examine paired stereoscopic radiographs and to see detail with a clarity not possible with conventional $x$-rays. In particular, the posterior elements of the spine can be seen more clearly. This technique has enabled us to identify small fractures in the laminae and apophyseal processes in patients who would otherwise be labelled as having 'nonspecific back pain'. The clinical histories, investigation results, and progress of these patients are here presented and the possible significance of these radiological changes is discussed.

\section{Method}

The 7 patients were part of a series of 200 patients either referred to the orthopaedic clinic or referred

Accepted for publication September 22, 1977

Correspondence to Professor M. I. V. Jayson, Department of Rheumatology, University of Manchester, Hope Hospital, Salford, Manchester M6 8HD by their general practitioner for a radiograph of the lumbar spine because of back pain. After clinical ${ }^{N}$

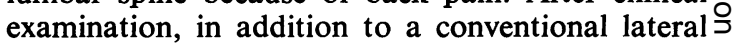
radiograph, paired anteroposterior (AP) radio- $\vec{c}$ graphs were taken with an axial shift in $x$-ray tube $\stackrel{\complement}{=}$ position to allow for stereoscopic viewing.

\section{Case reports}

\section{CASE 1}

A 30-year-old male motor mechanic, whose job required heavy and awkward lifting, had slipped 20 years previously on grease and developed acute low $\frac{\mathrm{D}}{\circ}$ back pain. This resolved with 2 months of bed rest $\stackrel{\varrho}{\longrightarrow}$ and local heat, but minor episodes recurred lasting $1 \overrightarrow{\overrightarrow{0}}$ or 2 days. 6 months previously, while lifting a heavy axle, he felt his back 'go'. Severe low back pain developed, radiating to the lateral aspect of the right thigh, which was made worse by bending, laughing, and sneezing. Physiotherapy with heat, mobilisation, traction, and exercises made the symptoms worse. Improvement was gradual over several weeks with rest.

Clinical examination in the acute phase showed a reduced lumbar lordosis with marked limitation of flexion, extension, and lateral flexion. Straight leg $\frac{D}{0}$ raising was $50^{\circ}$ on the right and $80^{\circ}$ on the left. There was some pain on internal rotation and flexion of the $\mathcal{N}$ right hip, but the range of movements was full. $N$ There were no neurological abnormalities.

Conventional AP and lateral radiographs showed no abnormality. However, on stereoscopic radiographs there was narrowing and osteophytosis of the right $L 4 / 5$ apophyseal joint. The superior facet of this joint was fractured and seen as a separate fragment. At follow-up 2 months later, when his symptoms had improved, repeat stereoscopic radiographs showed that the fragment had reunited with the superior facet, but the joint was still narrowed with osteophytes as before. 


\section{CASE 2}

A 35-year-old male electrician had an 11-year history of pain in the lumbar spine which had developed when he was performing heavy work in an enclosed space and unable to straighten up. Treatment with manipulation and a corset had provided only temporary relief. 5 months before presenting he had descended awkwardly from some scaffolding and developed acute pain in the low back, buttocks, and thighs with paraesthesiae which intensified with walking, bending, and lifting when tired, and was relieved by bed rest. Local heat provided some relief. Examination showed him to be a heavily built man with a reduced lumbar lordosis and marked loss of flexion and extension of the lumbar spine. Lateral flexion and rotation were normal. Straight leg raising was $75^{\circ}$ on both sides.

Standard AP and lateral radiographs showed no abnormality, but stereoscopic radiographs showed a narrowed left L4/5 apophyseal joint with a loose fragment of bone, and narrowed L2/3 apophyseal joints on both sides. Repeat stereoscopic radiographs, 5 months later when his symptoms improved, showed no evidence of a separate fragment and were consistent with healing although there was increased impingement and narrowing of the left L4/5 apophyseal joints.

\section{CASE 3}

A 42-year-old male baker, who had lifted bags of flour weighing 110-140 lbs (50-63.5 kg) for 27 years had a 10-year history of intermittent attacks of low back pain lasting 7 to 10 days. The attacks occurred every 3 to 4 months and would develop after relatively trivial stress. The pain was made worse by sitting, bending, walking, coughing, and sneezing.

An episode of low back pain radiating to the anterior aspect of the thighs developed suddenly. Examination showed loss of lumbar lordosis with a scoliosis to the right. All lumbar movements were very limited and right hip flexion and internal and external rotation were reduced because of pain. Straight leg raising on both sides was $75^{\circ}$. There were no other neurological abnormalities. He was treated with Maitland's mobilisation of the lumbar spine and symptoms remitted rapidly.

Standard AP and lateral radiographs showed narrowing of the L3/4 and L5/S1 disc spaces with some impingement of the L5/S1 apophyseal joints. Stereoscopic radiographs showed the changes in the L5/S1 apophyseal joints more clearly. There were degenerative changes of the right $L 3 / 4$ and $L 4 / 5$ apophyseal joints, and a large, loose fragment of bone adjacent to the inferior facet of L3. Repeat stereoscopic radiographs 8 months later showed similar changes with the loose bone fragment still present, and the left L3/4 apophyseal joint showing degenerative changes.

CASE 4

A 74-year-old housewife had a 7-year history of low back pain which had worsened over the previous 3 years. She suffered continuous lumbar pain with radiation to both buttocks which intensified with bending, sitting, and climbing stairs. Physiotherapy with traction, mobilisation, and exercises aggravated the symptoms.

Examination showed her to be overweight with a good posture. There was some loss of lumbar spine flexion, extension, and lateral flexion and rotation to the right. Straight leg raising on both sides was $70^{\circ}$ and there was no neurological abnormality.

Conventional AP and lateral radiographs showed disc narrowing at $L 2 / 3$ and $L 3 / 4$ levels, with a grade I spondylolisthesis of L5 on S1, and degenerative changes in the L4/5 and L5/S1 apophyseal joints. Stereoscopic radiographs showed much more clearly the degenerative changes of the L4/5 and L5/S1 apophyseal joints, with marked narrowing of the joint spaces, osteophytes, and impingement of the apophyseal processes on one another. There was also a fracture of the lamina of L5 on the right, with marked surrounding callus formation. Repeat radiographs 21 months later still showed the healing fracture, but with further degenerative changes of the L3/4 apophyseal joints.

\section{CASE 5}

A 51-year-old male transport driver, who had undertaken heavy loading and unloading, had had back pain intermittently since the age of 14 . The most recent attack had lasted for 21 months and had developed 3 months after a minor injury to the spine. He had acute central lumbosacral pain without radiation, aggravated by sitting or standing still and eased when lying. Short-wave diathermy gave considerable but temporary relief.

Examination showed him to be overweight with a normal posture. Flexion of the lumbar spine was reduced, but extension and lateral flexion and rotation were normal. Straight leg raising was $80^{\circ}$ on both sides and there was no neurological abnormality.

Standard AP and lateral radiographs showed a scoliosis concave to the right with disc-space narrowing at the L4/5 and L5/S1 levels, and narrowed apophyseal joints at L4/5 and L5/S1 on the left. Stereoscopic radiographs showed marked degenerative changes in the apophyseal joints at $\mathrm{L} 3 / 4, \mathrm{~L} 4 / 5$, and $\mathrm{L} 5 / \mathrm{S} 1$ levels, and at $\mathrm{L} 5 / \mathrm{S} 1$ on the right a fracture of the inferior facet with a large separate fragment of bone. 15 months later repeat radiographs showed no change, with the facetal fracture still present. 


\section{CASE 6}

A 56-year-old male clerk had a 20-year history of continuous pain in the lumbar, thoracic, and cervical spines, radiating to the thighs and calves, with acute exacerbations. Symptoms worsened when sitting and walking and were relieved by lying flat. He had had various forms of physiotherapy, epidural and local steroid injections, and many spinal corsets with no relief.

Examination showed a straight lumbar spine, with a marked upper lumbar lordosis. The spine was tender on palpation over L4 and L5. There was marked limitation of flexion associated with a scoliosis to the right. Extension, lateral flexion, and lateral rotation were also limited. Straight leg raising was $75^{\circ}$ on the right and $65^{\circ}$ on the left. There was no other neurological abnormality.

Standard AP and lateral radiographs showed a mild scoliosis concave to the right. There was discspace narrowing at $\mathrm{L} 3 / 4, \mathrm{~L} 4 / 5$, and $\mathrm{L} 5 / \mathrm{S} 1$ with a spondylolisthesis of $\mathrm{L4}$ on $\mathrm{L5}$, retrolisthesis of L5 on S1, and degenerative changes in the right L4/5 apophyseal joint. Stereoscopic radiographs showed the degenerative changes affecting the apophyseal joints at the lower 3 lumbar levels and a fracture of the inferior facet of L4 on the right. Repeat radiographs 16 months later showed that the fracture had healed with marked callus formation. The left L4/5 apophyseal joint had developed not only marked degenerative changes, but also a new fracture of the inferior facet. This new fracture could also be seen on a left oblique radiograph.

\section{CASE 7}

A 25-year-old male musician and ordinand had suffered recurrent episodes of low back pain lasting 1 to 2 days, starting at the age of 12 , and usually developing after playing the piano for several hours. His present attack had started one year previously with acute pain in the back and paraesthesiae in the anterolateral aspect of the left thigh and dorsum of the foot. Sitting, standing, and bending worsened the pain which was relieved by lying flat. Physiotherapy and a plaster jacket aggravated his symptoms.

Examination showed loss of lumbar lordosis, with restriction of flexion and extension, normal lateral flexion and rotation, and tenderness over the spines of L4 and L5. Straight leg raising was $60^{\circ}$ on the right and $45^{\circ}$ on the left. There was no other neurological abnormality.

Standard AP and lateral radiographs showed narrowing of the $L 4 / 5$ and $L 5 / S 1$ disc spaces. A large loose fragment of bone adjacent to the right inferior facet of L3 (Fig.) could be seen more clearly on the stereoscopic radiographs. There were also degenerative changes affecting the $\mathrm{L} 4 / 5$ and $\mathrm{L} 5 / \mathrm{S} 1$

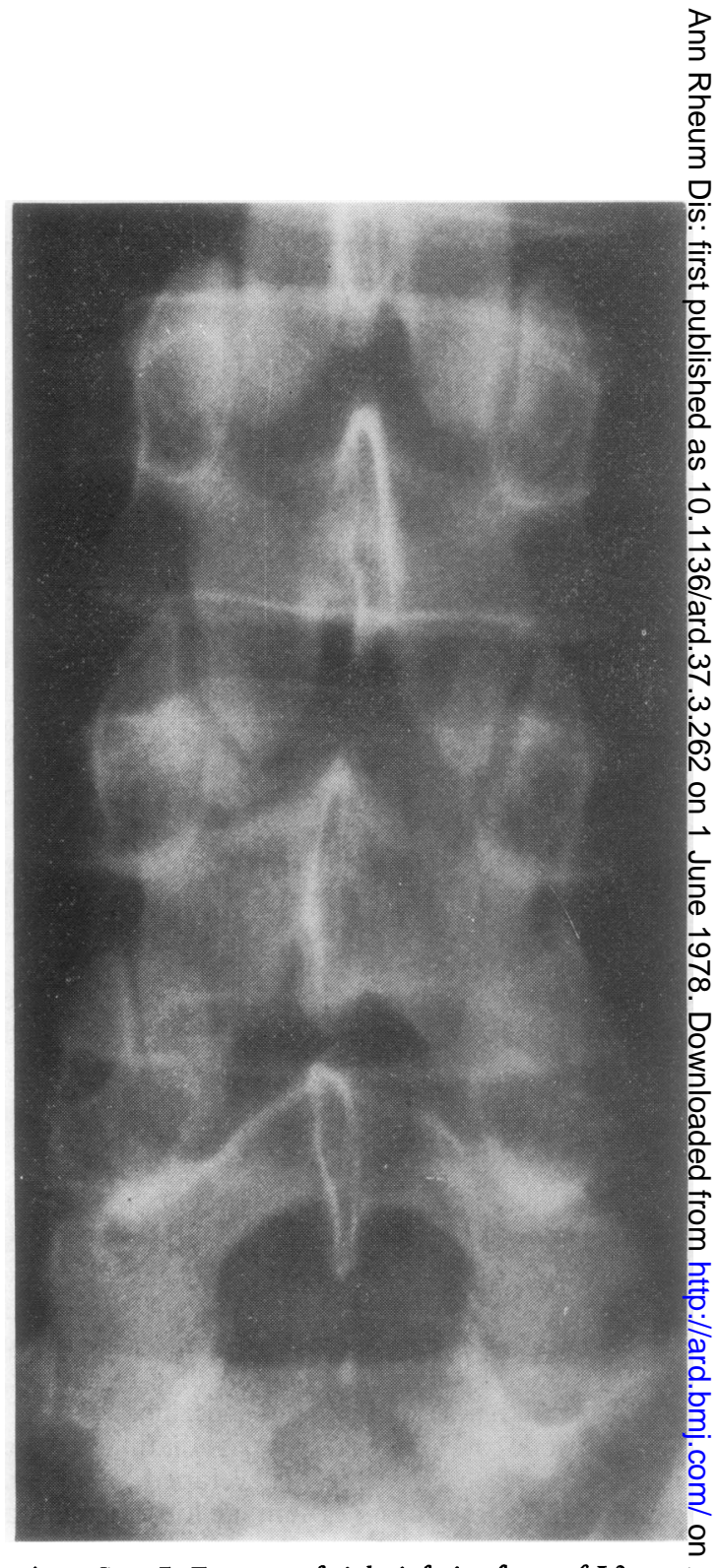

Fig. Case 7. Fracture of right inferior facet of L3.

apophyseal joints on both sides. Repeat radiơ graphs 11 months later, when he was still suffering from recurrent attacks of back pain, showed thes fracture still present. In the $L 4 / 5$ and $L 5 / S \Phi$ apophyseal joints, the degenerative changes were more advanced with subluxation and impingemen

\section{Discussion}

Gross tauma to the spine frequently causes fracture⿻ commonly to the vertebral bodies and these ar: readily identified by conventional radiologes Although it is possible that trauma could cause 
fractures of the posterior elements of the spine there was no clear history of such injuries in these patients. It is more likely that these small fractures were due to repeated minor stresses in the bone with eventual fatigue failure in the laminae and apophyseal facets. Wiltse et al. (1975) believe that such fatigue fractures are responsible for most cases of spondylolysis and spondylolytic spondylolisthesis. Shah et al. (1976; 1978) have shown that there is a greater concentration of strain in the posterior elements of the spine than in the anterior elements and that these strains are increased by extension. The areas of strain concentration correspond closely with the sites of fractures found in these patients. Experimentally, Farfan (1973) showed that axial compression loading could lead to fractures of the lamina and inferior articular process particularly if the joints are degenerate. These fractures resemble those described in this paper.

Radiographic examination of the pedicles, laminae, and apophyseal processes is notoriously difficult and it is not surprising that small fractures are difficult to recognise. Of these 7 patients, the fracture was found on conventional AP and lateral radiographs in only one and on oblique radiography in one other. In most the fracture was only found by careful examination using the stereoscopic technique. Undoubtedly, there are many patients with small fractures which we cannot detect at the present time.

It is difficult to evaluate the general significance of these small fractures. In some, healing occurred coincidentally with resolution of the back pain. But in others the fractures had failed to unite at follow-up and the pain pursued a variable course. In most of these patients there were degenerative changes in the adjacent apophyseal joints and intervertebral discs. It is impossible to weigh the relative contributions of the different factors to the back pain syndrome. In a broad comparison of the symptoms in these 7 patients and in a large group of back pain patients we could not identify any specific features enabling such fractures to be recognised clinically. It is notable that active physiotherapy usually exacerbated the symptoms.

The Zeiss stereoplotter was purchased with the aid of a grant from the Back Pain Association. This work was supported by grants from the Department of Health and the Arthritis and Rheumatism Council.

\section{References}

Farfan, H. F. (1973). Mechanical Disorders of the Low Back. Lea and Febiger, Philadelphia.

Jacoby, R. K., Sims-Williams, H., Jayson, M. I. V., and Baddeley, H. (1976). Radiographic stereoplotting: a new technique and its application to the study of the spine. Annals of the Rheumatic Diseases, 35, 168-170.

Shah, J. S., Coggins, J., Rogers, R., Jayson, M. I. V., and Hampson, W. G. J. (1976). Surface strain distribution in isolated single lumbar vertebrae. Annals of the Rheumatic Diseases, 35, 51-55.

Shah, J. S., Hampson, W. G. J. and Jayson, M. I. V. (1978). Surface strain distribution in the cadaveric lumbar spine. Journal of Bone and Joint Surgery (in press).

Vernon-Roberts, B., and Pirie, C. J. (1973). Healing trabecular microfractures in the bodies of lumbar vertebrae. Annals of the Rheumatic Diseases, 32, 406-412.

Wiltse, L. L., Widdell, E. H., and Jackson, D. W. (1975). Fatigue fracture: the basic lesion in isthmic spondylolisthesis. Journal of Bone and Joint Surgery, 57A, 17-22. 